\title{
The Endocannabinoid System across Postnatal Development in Transmembrane Domain Neuregulin 1 Mutant Mice
}

\author{
Rose Chesworth ${ }^{1 * t}$, Leonora E. Long ${ }^{2,3+}$, Cynthia Shannon Weickert ${ }^{2,3,4 \ddagger}$ and Tim Karl ${ }^{1,2,3 * \neq}$ \\ ${ }^{1}$ School of Medicine, Western Sydney University, Campbelltown, NSW, Australia, ${ }^{2}$ Schizophrenia Research Institute, Sydney, \\ NSW, Australia, ${ }^{3}$ Neuroscience Research Australia, Randwick, NSW, Australia, ${ }^{4}$ School of Psychiatry, University of New \\ South Wales, Sydney, NSW, Australia
}

\section{OPEN ACCESS}

Edited by:

Stefan Borgwardt,

University of Basel, Switzerland

Reviewed by:

Steven R. Laviolette,

University of Western

Ontario, Canada

Dragos Inta,

Zentralinstitut für Seelische

Gesundheit, Germany

*Correspondence:

Rose Chesworth

r.chesworth@westernsydney.edu.au;

Tim Karl

t.karl@westernsydney.edu.au

tThese authors have contributed equally to this work.

fShared last authors.

Specialty section:

This article was submitted to

Schizophrenia,

a section of the journal

Frontiers in Psychiatry

Received: 22 June 2017

Accepted: 15 January 2018

Published: 07 February 2018

Citation:

Chesworth R, Long LE, Weickert CS and Karl T (2018) The

Endocannabinoid System across Postnatal Development

in Transmembrane Domain

Neuregulin 1 Mutant Mice.

Front. Psychiatry 9:11

doi: 10.3389/fpsyt.2018.00011
The use of cannabis is a well-established component risk factor for schizophrenia, particularly in adolescent individuals with genetic predisposition for the disorder. Alterations to the endocannabinoid system have been found in the prefrontal cortex of patients with schizophrenia. Thus, we assessed whether molecular alterations exist in the endocannabinoid signalling pathway during brain development in a mouse model for the schizophrenia risk gene neuregulin 1 (Nrg1). We analysed transcripts encoding key molecules of the endocannabinoid system in heterozygous transmembrane domain Nrg1 mutant mice (Nrg1 TM HET), which is known to have increased sensitivity to cannabis exposure. Tissue from the prelimbic cortex and hippocampus of male and female Nrg1 TM HET mice and wild type-like littermates was collected at postnatal days (PNDs) 7, 10, 14, 21, 28, 35, 49, and 161. Quantitative polymerase chain reaction was conducted to assess mRNA levels of cannabinoid receptor $1\left(\mathrm{CB}_{1} \mathrm{R}\right)$ and enzymes for the synthesis and breakdown of the endocannabinoid 2-arachidonoylglycerol [i.e., diacylglycerol lipase alpha (DAGL $\alpha$ ), monoglyceride lipase (MGLL), and $\alpha / \beta$-hydrolase domain-containing 6 (ABHD6)]. No sex differences were found for any transcripts in either brain region; thus, male and female data were pooled. Hippocampal and cortical mRNA expression of DAGL $\alpha$, MGLL, and ABHD6 increased until PND 21-35 and then decreased and stabilised for the rest of postnatal development. Hippocampal $\mathrm{CB}_{1} \mathrm{R}$ mRNA expression increased until PND 21 and decreased after this age. Expression levels of these endocannabinoid markers did not differ in Nrg1 TM HET compared to control mice at any time point. Here, we demonstrate dynamic changes in the developmental trajectory of several key endocannabinoid system transcripts in the mouse brain, which may correspond with periods of endocannabinoid system maturation. Nrg1 TM HET mutation did not alter the developmental trajectory of the endocannabinoid markers assessed, suggesting that other mechanisms may be responsible for the exaggerated cannabinoid susceptibility in these mice.

Keywords: schizophrenia, endocannabinoid system, neuregulin 1, development, cannabinoid receptor 1, diacylglycerol lipase alpha, monoglyceride lipase, $\alpha / \beta$-hydrolase domain-containing 6

Abbreviations: 2-AG, 2-arachidonoylglycerol; ABHD6, alpha/beta-hydrolase domain-containing 6; AEA, $\mathrm{N}$-arachidonoylethanolamine; ANOVA, analysis of variance; $\mathrm{CB}_{1} \mathrm{R}$, cannabinoid receptor 1; cDNA, complementary deoxyribonucleic acid; DAGL $\alpha$, diacylglycerol lipase alpha; DLPFC, dorsolateral prefrontal cortex; MGLL, monoglyceride lipase; mRNA, messenger ribonucleic acid; Nrg1 TM HET, Neuregulin 1 transmembrane domain heterozygous; PND, postnatal day; qPCR, quantitative polymerase chain reaction; RIN, RNA integrity number; RNA, ribonucleic acid; SEM, standard error of the mean; WT, wild type. 


\section{INTRODUCTION}

Schizophrenia is a chronic, debilitating mental disorder that can develop through interactions between genetic susceptibility and environmental insults (1-3). The use of cannabis can increase the risk of developing schizophrenia $(4,5)$, and usage rates are elevated in individuals who carry genetic risk alleles for the disorder (6). Furthermore, a single-nucleotide polymorphism associated with cannabis dependence is located in the neuregulin 1 (NRG1) gene, which is a susceptibility gene for schizophrenia $(7,8)$. NRG1 and its cognate receptor ErbB4 regulate the development of activitydriven glutamatergic synapse development (9), indicating that NRG1 signalling may contribute to schizophrenia pathophysiology in the context of the glutamate hypothesis of schizophrenia. While the mechanisms by which cannabis exposure and genetic factors interact to bring about psychotic illness are unclear, it is possible that genetically induced dysregulation of endogenous cannabinoid signalling may be a contributing factor (10).

The endogenous cannabinoid (endocannabinoid) system is constituted by endogenous receptors [e.g., cannabinoid receptor $1\left(\mathrm{CB}_{1} \mathrm{R}\right)$ and cannabinoid receptor 2] and ligands that regulate neurotransmission and govern neurodevelopment [e.g., 2-arachidonoylglycerol (2-AG) and $\mathrm{N}$-arachidonoylethanolamine] (11). Importantly, the endocannabinoid system appears to undergo changes in patients with schizophrenia. Elevated levels of anandamide, one of the main brain endocannabinoids, are present in the cerebrospinal fluid of patients with schizophrenia (12) and are inversely associated with psychotic symptoms in treatment naive patients (13). Also, mRNA expression of the predominant brain cannabinoid receptor, which mediates the psychoactive effects of cannabis, $\mathrm{CB}_{1} \mathrm{R}$, is reduced in the dorsolateral prefrontal cortex (DLPFC) of patients with schizophrenia $(14,15)$. In contrast, we have also found elevated $\mathrm{CB}_{1} \mathrm{R}$ binding in the DLPFC of people with paranoid schizophrenia (16). Thus, is possible that elevated risk for developing schizophrenia following cannabis exposure may result from altered endocannabinoid signalling. Although the nature of the change to the endocannabinoid system found in adults is unclear, it is possible that the development of the endocannabinoid system could be altered in schizophrenia either as an underling disruption or occurring in response to the disease. In line with this is the fact that the endocannabinoid system undergoes significant changes around the typical time of onset of schizophrenia (e.g., adolescence to early adulthood) (17).

We sought to address two aims: (1) to chart the developmental trajectory of mRNAs involved with the endocannabinoid system in murine brain and (2) to determine whether developmental changes in the endocannabinoid signalling system may occur as a direct response to genetic susceptibility to schizophrenia. We did this by examining two schizophrenia-relevant brain regions across postnatal development (i.e., the prelimbic cortex and the hippocampus) in an established mouse model for schizophrenia, the heterozygous transmembrane domain Nrg1 mutant mice (Nrg1 TM HET). Nrg1 TM HET mice display construct validity for schizophrenia, as schizophrenia patients have been found to exhibit a missense mutation in the transmembrane domain of NRG1 (18). Nrg1 TM HET mice also exhibit face and predictive validity for schizophrenia, as they display behavioural features relevant to the symptoms of schizophrenia (19-22), some of which can be ameliorated by antipsychotic treatment (7). Importantly, Nrg1 TM HET mice also exhibit altered susceptibility to the behavioural and neural effects of cannabinoids (23-28), which is relevant to the increased cannabis sensitivity observed in patients with schizophrenia (29).

Previous work in our laboratory demonstrated region-specific changes in receptor binding for $\mathrm{CB}_{1} \mathrm{R}$ during adolescence and adulthood in $\mathrm{Nrg} 1 \mathrm{TM}$ HET mice $(10,28)$. However, these studies did not test the extent to which these changes may have preceded adolescence [analysis of brains from postnatal day (PND) 53 onwards only] and only examined $\mathrm{CB}_{1} \mathrm{R}$. This study examined the developmental trajectory of four endocannabinoid system transcripts from PND 7-161. We assessed mRNA expression of $\mathrm{CB}_{1} \mathrm{R}$, as well as of the enzymes diacylglycerol lipase alpha (DAGL $\alpha)$, monoglyceride lipase (MGLL), and $\alpha / \beta$-hydrolase domain-containing 6 (ABHD6). We chose these enzymes as they catalyse the synthesis and hydrolysis of the most abundant brain endocannabinoid, 2-AG. We quantified mRNA in the hippocampus and prelimbic cortex because these regions show high levels of $\mathrm{CB}_{1} \mathrm{R}$ expression (30) and are critically implicated in schizophrenia $(31,32)$. We hypothesised that there would be region-specific dynamic changes in $\mathrm{CB}_{1} \mathrm{R}, \mathrm{DAGL} \alpha$, MGLL, and ABHD6 mRNA across development. We also hypothesised that the mRNA expression of endocannabinoids would be different in Nrg1 TM HET mice compared to control mice-notably that the expression of these markers may be elevated in Nrg1 TM HET mice or may occur earlier in mutant mice compared to control littermates-and that these differences may account for the altered response of Nrg1 TM HET mice to cannabinoids (33).

\section{MATERIALS AND METHODS}

\section{Animals}

Male and female heterozygous $\mathrm{Nrg1} \mathrm{TM}^{+/-}$mice (Nrg1 TM HET) and $\mathrm{Nrg} \mathrm{TM}^{+/+}$[wild type (WT)] littermates were bred as described previously (19) and backcrossed for $>10$ generations onto a C57BL6/JArc background. Mice were bred and housed at Australian BioResources (Moss Vale, Australia). Mice were housed with their dams in litters prior to weaning at PND 21, where they were group housed (2-4 animals per cage) in individually ventilated cages (Type Mouse Version 1; Airlaw, Smithfield, Australia; air change: 90-120 times per hour averaged; air speed: $0.12 \mathrm{~m} / \mathrm{s}$; passive exhaust ventilation system). Cages contained a wire hopper, giving the animals some limited vertical climbing opportunities, and also contained a mouse igloo (Bioserv, Frenchtown, USA) and tissues for nesting material. Mice were kept under a 12:12 h light:dark schedule, and food and water were available ad libitum. Tissue was collected from 5 to 11 litters per age and sex group. Genotypes were confirmed after weaning using tail-tip biopsy and polymerase chain reaction (PCR) amplification (primers: forward 5'-GCTAGCTTGTTATTTATGCTTAAAG-3'; WT reverse 5'-CCACCACACACATGATGCCGAC-3'; Nrg1 TMHET reverse $5^{\prime}$-GCACAGTCGAGGCTGATCAGCG-3'). Research and animal care procedures were approved by the University of New South Wales Animal Care and Ethics Committee (ACEC) in 
accordance with the Australian Code of Practice for the Care and Use of Animals for Scientific Purposes (ACEC approval number: $10 / 98 \mathrm{~B})$. The protocol was approved by the University of New South Wales ACEC.

\section{Tissue Preparation and RNA Extraction}

Total RNA was extracted for quantitative PCR (qPCR) analysis from the left hippocampus (4.3-18.3 mg, $N=181$ ) and prelimbic cortex (1.3-11.7 mg, $N=147)$ of male and female Nrg1 TM HET and WT littermates at PND 7, 10, 14, 21, 28, 35, and 161 (Table 1) using TRIzol following the manufacturer's protocol (Invitrogen, Carlsbad, CA, USA). Total RNA was suspended in DEPC-treated water after purification by precipitation (Sigma-Aldrich, Castle Hill, NSW, Australia). The yield of total RNA was analysed using a spectrophotometer (Nanodrop ND-1000; Thermo Scientific, Wilmington, DE, USA). The quality of total RNA was determined using an Agilent Bioanalyzer 2100 (Agilent Technologies, Palo Alto, CA, USA): 100-200 ng RNA was applied to an RNA 6000 Nano LabChip, without heating prior to loading. The RNA integrity number (RIN) was used as an indicator of RNA quality, ranging from 1 to 10 (lowest to highest quality). Samples had an average RIN of $9.03 \pm 0.10$ (Table 1 ). Any sample with a $\mathrm{RIN} \leq 6.5$ was excluded from qPCR experiments.

\section{Reverse Transcription and qPCR}

Complementary deoxyribonucleic acid (cDNA) was synthesised in two reactions of $0.5-2 \mu \mathrm{g}$ of total RNA using the Superscript III First-Strand Synthesis Kit (Invitrogen) according to the manufacturer's protocol. Predesigned TaqMan Gene Expression Assays (Applied Biosystems, Foster City, CA, USA) were chosen for genes of interest and for three housekeeper control transcripts (Table 2). qPCR was performed with an ABI Prism 7900HT Fast real-time PCR system with a 384-well format. The PCR reaction was initiated by uracil-DNA glycosylase treatment for $2 \mathrm{~min}$ at

TABLE 1 | Summary of developmental cohort used for experiments.

\begin{tabular}{|c|c|c|c|c|}
\hline Postnatal day & Genotype & Gender & RIN & $n$ \\
\hline \multicolumn{5}{|l|}{ Hippocampus } \\
\hline 7 & 12 WT, 10 Nrg1 TM HET & $11 \mathrm{~F}, 11 \mathrm{M}$ & $9.63 \pm 0.47$ & 22 \\
\hline 10 & 12 WT, 12 Nrg1 TM HET & $12 \mathrm{~F}, 12 \mathrm{M}$ & $9.70 \pm 0.35$ & 24 \\
\hline 14 & 12 WT, 11 Nrg1 TM HET & $12 \mathrm{~F}, 11 \mathrm{M}$ & $9.24 \pm 0.33$ & 23 \\
\hline 21 & 12 WT, 12 Nrg1 TM HET & $12 \mathrm{~F}, 12 \mathrm{M}$ & $9.10 \pm 0.25$ & 24 \\
\hline 28 & 12 WT, 12 Nrg1 TM HET & $12 \mathrm{~F}, 12 \mathrm{M}$ & $8.88 \pm 0.27$ & 24 \\
\hline 35 & 12 WT, 11 Nrg1 TM HET & $12 \mathrm{~F}, 11 \mathrm{M}$ & $8.73 \pm 0.36$ & 23 \\
\hline 49 & 10 WT, 11 Nrg1 TM HET & $11 \mathrm{~F}, 10 \mathrm{M}$ & $8.73 \pm 0.46$ & 21 \\
\hline 161 & 10 WT, 10 Nrg1 TM HET & $9 \mathrm{~F}, 11 \mathrm{M}$ & $8.77 \pm 0.46$ & 20 \\
\hline
\end{tabular}

Prelimbic cortex

\begin{tabular}{llcll}
\hline 7 & $9 \mathrm{WT}, 7 \mathrm{Nrg} 1 \mathrm{TM} \mathrm{HET}$ & $9 \mathrm{~F}, 7 \mathrm{M}$ & $9.61 \pm 0.54$ & 16 \\
10 & $9 \mathrm{WT}, 7 \mathrm{Nrg} 1 \mathrm{TM} \mathrm{HET}$ & $8 \mathrm{~F}, 8 \mathrm{M}$ & $9.48 \pm 0.57$ & 15 \\
14 & $9 \mathrm{WT}, 8 \mathrm{Nrg} 1 \mathrm{TM} \mathrm{HET}$ & $10 \mathrm{~F}, 7 \mathrm{M}$ & $9.30 \pm 0.53$ & 17 \\
21 & $9 \mathrm{WT}, 7 \mathrm{Nrg1}$ TM HET & $9 \mathrm{~F}, 7 \mathrm{M}$ & $8.61 \pm 1.09$ & 16 \\
28 & $12 \mathrm{WT}, 11 \mathrm{Nrg}$ TM HET & $11 \mathrm{~F}, 12 \mathrm{M}$ & $8.75 \pm 0.58$ & 23 \\
35 & $12 \mathrm{WT}, 10 \mathrm{Nrg1}$ TM HET & $11 \mathrm{~F}, 11 \mathrm{M}$ & $8.60 \pm 0.76$ & 22 \\
49 & $9 \mathrm{WT}, 8 \mathrm{Nrg1}$ TM HET & $7 \mathrm{~F}, 10 \mathrm{M}$ & $8.55 \pm 0.51$ & 17 \\
161 & $11 \mathrm{WT}, 9 \mathrm{Nrg} 1 \mathrm{TM} \mathrm{HET}$ & $9 \mathrm{~F}, 11 \mathrm{M}$ & $8.81 \pm 0.31$ & 20
\end{tabular}

F, female; $M$, male; $n$, number of mice per postnatal age group; RIN, RNA integrity number. $50^{\circ} \mathrm{C}$ and denaturation for $10 \mathrm{~min}$ at $95^{\circ} \mathrm{C}$, followed by 40 cycles consisting of heating to $95^{\circ} \mathrm{C}$ for $15 \mathrm{~s}$ followed by annealing and extension at $60^{\circ} \mathrm{C}$ for $1 \mathrm{~min}$. Measurements were performed in duplicate and relative quantities determined from a seven-point standard curve. Control wells containing no cDNA template displayed no amplification. Efficiencies of the qPCR reactions ranged from 61 to $92 \%$, and $r^{2}$ values were between 0.96 and 1.00 . Outliers were excluded from qPCR analysis if their normalised expression values were greater than $2.5 \mathrm{SD}$ from the group mean. Expression levels were normalised to the geometric mean of three reference genes (Table 2).

\section{Statistical Analysis}

Quantitative PCR data were analysed using SPSS Statistics 22 (IBM, NY, USA). Differences in normalised mRNA expression were assessed with three-way analysis of variance (ANOVA) with "genotype" (WT/Nrg1 TM HET), "sex" (male/female), and "age" (PND 7, 10, 14, 21, 28, 35, 49, and 161) as between factors. Polynomial contrasts were used to identify linear, quadratic, and cubic trends in mRNA expression across PND ages. Data are presented as mean \pm SEM, and differences were regarded as statistically significant if $p<0.05$.

\section{RESULTS}

\section{Housekeeper Controls}

The expression of house-keeping genes was stable across postnatal development. The geometric mean of the expression of Ubc and Tbp mRNA and 18S rRNA did not change significantly over postnatal development in the hippocampus or prelimbic cortex (Figure S1 in Supplementary Material).

\section{Sex Differences}

Endocannabinoid mRNA expression did not differ between male and female mice in either brain region for any endocannabinoid marker investigated; thus, data were collapsed across sex (threeway ANOVA main effect of "sex": all $F<1$, all $p>0.05$ ).

\section{Cannabinoid Receptor 1}

Cannabinoid receptor 1 mRNA expression exhibited a trend for changes across development in the hippocampus, but not in the prelimbic cortex [main effect of "age": hippocampus $F(7,158)=2.0, p=0.06$; Figure 1A; prelimbic cortex

TABLE 2 | Applied Biosystems TaqMan gene expression assay numbers.

\begin{tabular}{|c|c|c|}
\hline Gene name & Gene symbol & Taqman assay \\
\hline \multicolumn{3}{|l|}{ Genes of interest } \\
\hline Cannabinoid $\mathrm{CB}_{1}$ receptor & Cnr1 & Mm00432621_s1 \\
\hline Diacylglycerol lipase alpha & Dagl $\alpha$ & Mm00813830_m1 \\
\hline Monoglyceride lipase & Mgll & Mm00449274_m1 \\
\hline$\alpha / \beta$-hydrolase domain containing 6 & Abhd6 & Mm00481199_m1 \\
\hline \multicolumn{3}{|l|}{ Reference genes } \\
\hline TATA box binding protein & Tbp & Mm00446973_m1 \\
\hline Ubiquitin C & Ubc & Mm01201237_m1 \\
\hline Eukaryotic 18S rRNA & $18 \mathrm{~S}$ & Hs99999901_s1 \\
\hline
\end{tabular}




\section{Hippocampus}
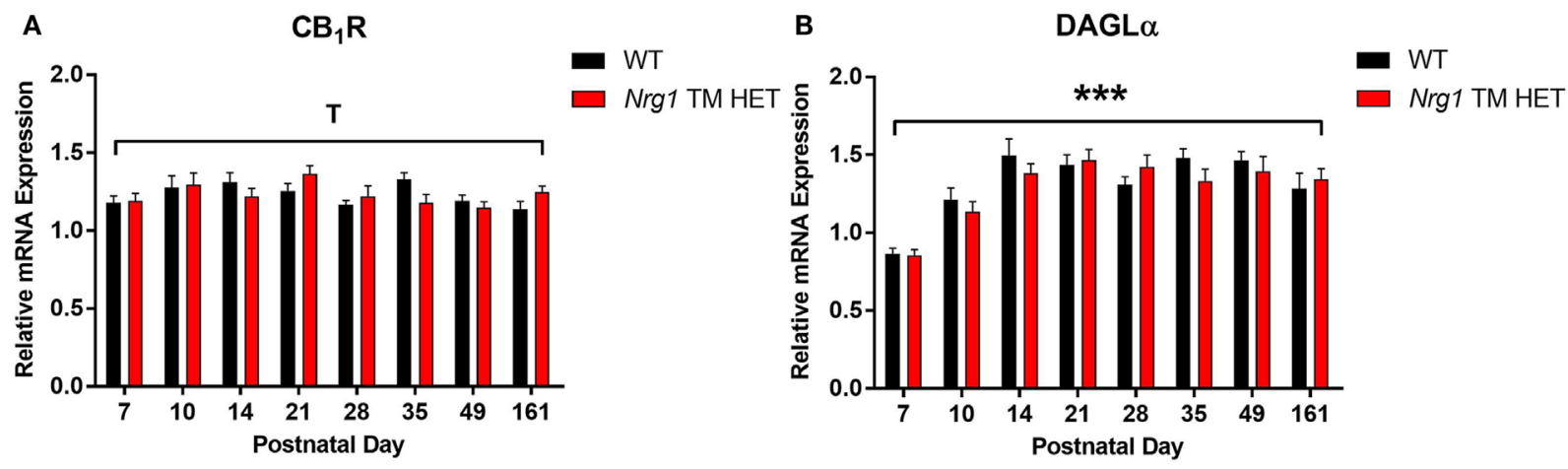

C

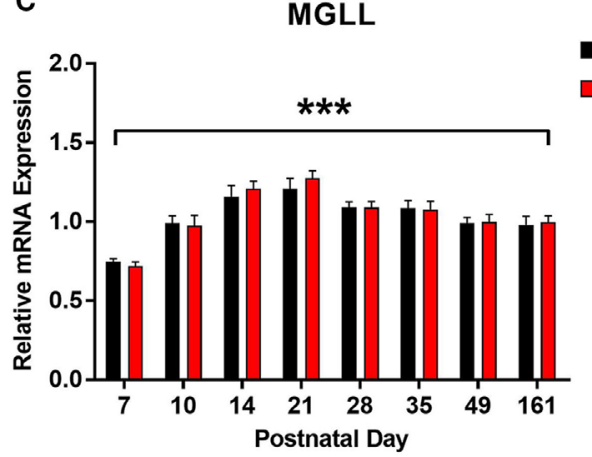

D

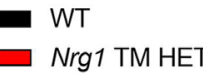

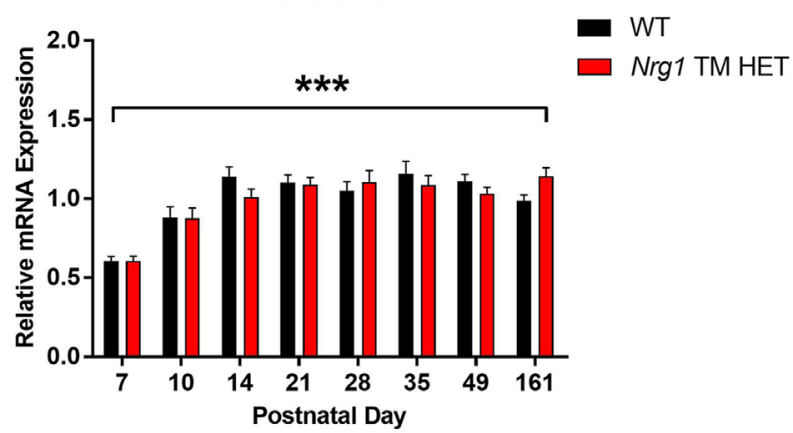

FIGURE 1 | Endocannabinoid mRNA expression in hippocampus of heterozygous transmembrane domain Nrg1 mutant mice (Nrg1 TM HET mice) and wild type (WT)-like controls. Expression of mRNA for (A) $\mathrm{CB}_{1}$ receptor ( $\left.\mathrm{CB}_{1} \mathrm{R}\right)$, (B) diacylglycerol lipase alpha (DAGL $\left.\alpha\right)$, (C) monoglycerol lipase (MGLL), and (D) $\alpha / \beta$-hydrolase domain-containing 6 (ABHD6) was determined by quantitative polymerase chain reaction $[y$-axis, mean $( \pm S E M)$ expression normalized to the geometric mean of three reference genes] and plotted by postnatal day. Main effects of "age" are indicated on graphs by asterisks $\left({ }^{\star \star \star} p<0.001\right)$; trends for effects of age are indicated by "T" (' $p=0.06)$.

$F(7,134)=1.6, p=0.10$; Figure 2A]. A significant quadratic contrast $(p=0.047)$ for hippocampal $\mathrm{CB}_{1} \mathrm{R}$ mRNA expression data indicates that overall $\mathrm{CB}_{1} \mathrm{R}$ expression increased until PND 21 and decreased after that age. There were no differences in $\mathrm{CB}_{1} \mathrm{R}$ mRNA expression between WT and Nrg1 TM HET mice in the hippocampus $[F(1,158)=0.01, p=0.90]$ or prelimbic cortex $[F(1,134)=0.1, p=0.80]$.

\section{Diacylglycerol Lipase Alpha}

Diacylglycerol lipase alpha mRNA expression changed significantly during postnatal development in the hippocampus [main effect of "age": $F(7,158)=15.5, p<0.001$; Figure 1B], with a significant cubic contrast $(p=0.004)$, indicating that DAGL $\alpha$ mRNA expression increased from PND 7 to 21, then decreased at PND 28, and stabilised for the rest of postnatal development. Hippocampal DAGL $\alpha$ mRNA expression was not different between WT and Nrg1 TM HET mice across postnatal development $[F(1,158)=0.60, p=0.50]$. There was no significant change in postnatal DAGL $\alpha$ mRNA expression across development in the prelimbic cortex overall $[F(7,134)=1.7, p=0.10]$; this was not different between WT and $N r g 1$ TM HET mice $[F(1,134)=1.2$, $p=0.30$; Figure 2B].

\section{Monoglyceride Lipase}

Monoglyceride lipase mRNA expression changed significantly during postnatal development in both the hippocampus and the prelimbic cortex [hippocampus: $F(7,158)=19.1, p<0.001$; prelimbic cortex: $F(7,135)=4.8, p<0.001]$. In the hippocampus, MGLL mRNA expression increased from PND 7 to 14 , then dropped, and remained stable until PND 35, before dropping to a lower level of expression at PND 49-161 (significant cubic contrast, $p<0.001$; Figure 1C). In the prelimbic cortex, MGLL mRNA expression increased in a linear fashion over postnatal development, reaching peak levels at PND 35 (significant linear contrast, $p<0.001$; Figure 2C). In both the hippocampus and the prelimbic cortex, MGLL mRNA expression across development was similar in WT and Nrg1 TM HET mice [hippocampus: $F(1,158)=0.2, p=0.70$; prelimbic cortex: $F(1,134)=1.4$, $p=0.20]$.

\section{$\alpha / \beta$-Hydrolase Domain-Containing 6}

$\alpha / \beta$-Hydrolase domain-containing 6 mRNA expression levels showed significant developmental changes in both brain regions investigated [hippocampus: $F(7,157)=19.6, p<0.001$; prelimbic cortex $F(7,132)=2.5, p=0.02]$. In the hippocampus, ABHD6 


\section{Prelimbic Cortex}
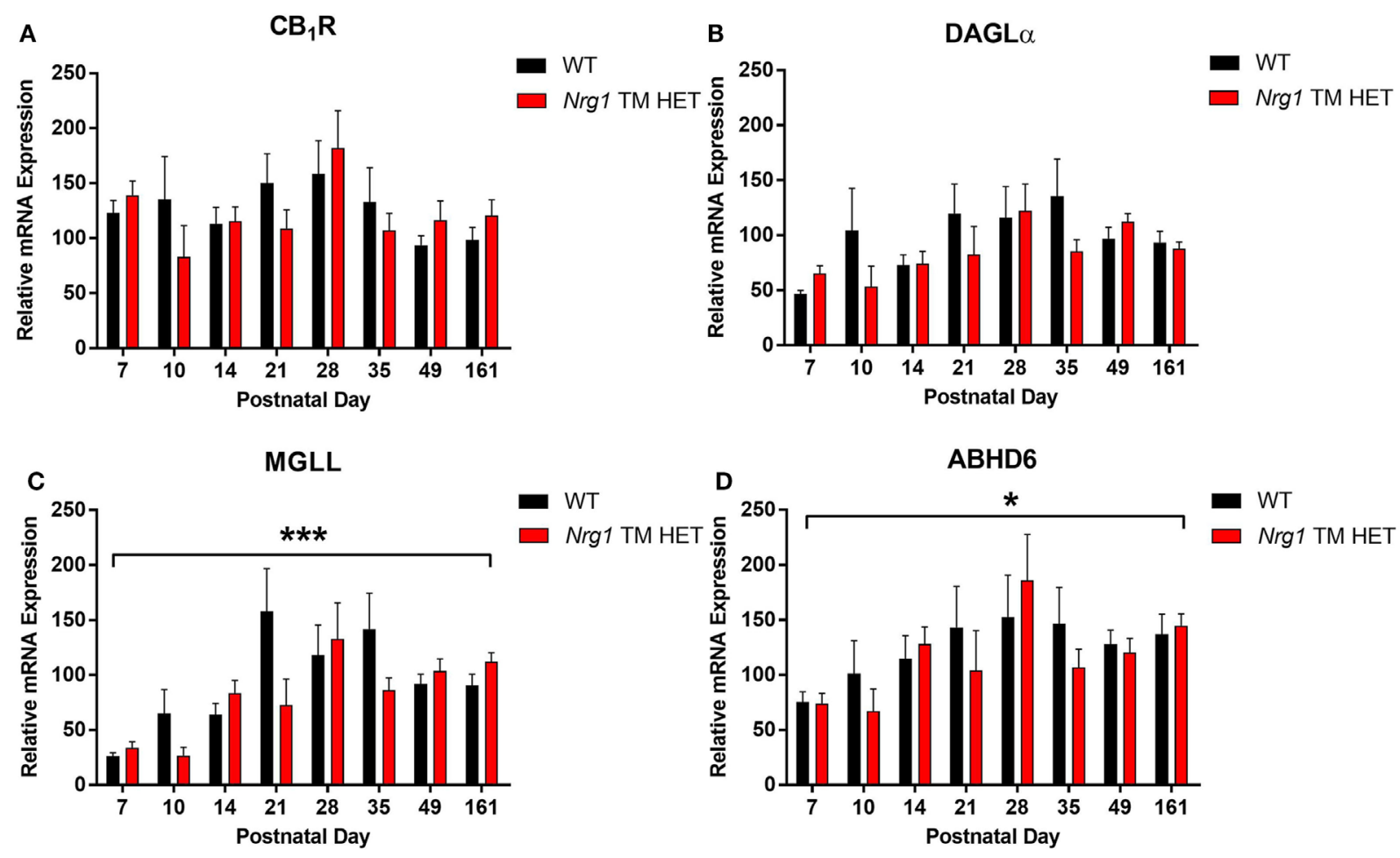

FIGURE 2 | Endocannabinoid mRNA expression in the prelimbic cortex of heterozygous transmembrane domain Nrg1 mutant mice (Nrg1 TM HET mice) and wild type (WT)-like controls. Expression of mRNA for (A) $C_{1}$ receptor $\left(C_{1} B_{1}\right)$, (B) diacylglycerol lipase alpha (DAGL $\left.\alpha\right)$, (C) monoglycerol lipase (MGLL), and (D) $\alpha / \beta$-hydrolase domain-containing 6 (ABHD6) was determined by quantitative polymerase chain reaction $[y$-axis, mean ( \pm SEM) expression normalized to the geometric mean of three reference genes] and plotted by postnatal day. Main effects of "age" are indicated on graphs by asterisks $\left({ }^{*} p<0.05\right.$, $\left.{ }^{\star \star \star} p<0.001\right)$.

mRNA expression slowly increased until PND 14-21, after which it stabilised (significant quadratic contrast, $p<0.001$; Figure 1D). In the prelimbic cortex, ABHD6 mRNA expression increased in a linear fashion over postnatal development until PND 28 (significant linear contrast, $p=0.006$; Figure 2D). ABHD6 mRNA expression was not different between WT and Nrg1 TM HET mice in either brain region investigated [hippocampus: $F(1,157)=0.10, p=0.70$; prelimbic cortex: $F(1,132)=0.40$, $p=0.50]$.

\section{DISCUSSION}

The present experiments describe in detail the postnatal development of the transcripts encoding $\mathrm{CB}_{1} \mathrm{R}$ and 2-AG metabolic enzymes DAGL $\alpha$, MGLL, and ABHD6 in control (WT) and transmembrane domain Nrg1 mutant mice. There was a trend for postnatal correlation of hippocampal $\mathrm{CB}_{1} \mathrm{R}$ mRNA with age, and an analysis of non-linear change over time detected increased hippocampal $\mathrm{CB}_{1} \mathrm{R}$ expression until PND 21. We found robust support for the developmental alterations in transcripts involved in the regulation of 2-AG in the hippocampus and prelimbic cortex. Expression of the enzymes catalysing 2-AG synthesis (DAGL $\alpha$ ) and hydrolysis (MGLL and ABHD6) increased markedly in the first few weeks of postnatal life, with peak levels being reached in the hippocampus at PND 14, about 2 weeks prior to the prefrontal cortex (PND 28-35). We did not find any evidence that inheriting mutant Nrg1 leads to altered endocannabinoid-related mRNA levels: Nrg1 TM HET mice displayed similar developmental trajectories of endocannabinoid markers as WT mice.

We found changes in $C_{1} R$ mRNA expression in the hippocampus across postnatal development: $\mathrm{CB}_{1} \mathrm{R}$ mRNA expression increased from PND 7 to 21 and decreased thereafter. A similar pattern of hippocampal $\mathrm{CB}_{1} \mathrm{mRNA}$ expression has been reported in rats; $\mathrm{CB}_{1}$ mRNA expression peaks in early postnatal development and decreases in adulthood (34). Our findings also correspond with reports using human hippocampal tissue; higher $\mathrm{CB}_{1}$ mRNA expression is present in fetal human hippocampal tissue (approximately 20 weeks into development) compared to adult hippocampal tissue (46-62 years old) (35). The increase in $\mathrm{CB}_{1} \mathrm{R}$ mRNA expression in early postnatal development may correspond with the regulation of neurotransmission by the endocannabinoid system in early life. The reduction in $\mathrm{CB}_{1} \mathrm{R}$ mRNA expression following PND 21 may be linked to the maturation of GABAergic hippocampal interneurons (36), which take over inhibitory presynaptic neurotransmission previously regulated by the endocannabinoid system (17). 
Prelimbic $\mathrm{CB}_{1} \mathrm{R}$ mRNA expression did not change across postnatal development. This may be because non-human cortical $\mathrm{CB}_{1} \mathrm{R}$ mRNA expression has been found to be more dynamic during embryonic than postnatal development. Rats exhibit highest $\mathrm{CB}_{1} \mathrm{R}$ mRNA ratios in the cerebral cortex during gestational and early postnatal development (PND 1 and PND 5) $(34,37)$, and in macaques, dynamic changes in DLPFC $\mathrm{CB}_{1} \mathrm{R}$ mRNA are detected predominantly during embryonic development (38). Thus, measuring mRNA expression from PND 7 onwards may have been too late to detect substantial changes in cortical $\mathrm{CB}_{1} \mathrm{mRNA}$ expression.

This study was not able to detect changes in $\mathrm{CB}_{1} \mathrm{R}$ mRNA expression in Nrg1 TM HET mice compared to WT littermates. Previous autoradiographic receptor binding studies in the same mouse line demonstrated decreased $\mathrm{CB}_{1} \mathrm{R}$ protein levels in the substantia nigra in adolescence (PND 53) and increased levels in adulthood (PND 98-140) $(10,28)$. However, $\mathrm{CB}_{1} \mathrm{R}$ binding in the medial prefrontal cortex and hippocampus in adult $\mathrm{Nrg} 1 \mathrm{TM}$ HET was not different to that of WT mice $(10,28)$, which is in line with our findings. This suggests region-specific changes in $\mathrm{CB}_{1} \mathrm{R}$ expression in $\mathrm{Nrg} 1 \mathrm{TM}$ HET mice. It is also possible that there are differences between the expression profiles of $\mathrm{CB}_{1} \mathrm{R}$ receptor protein and mRNA levels, similar to what has been observed in human schizophrenia DLPFC tissue $(39,40)$.

We observed dynamic changes in mRNA expression of DAGL $\alpha$, MGLL, and ABHD6 across postnatal development in both the hippocampus and the prelimbic cortex. Our study is the first to describe mouse ABHD6 mRNA expression across postnatal development; this has not been reported in rodents or non-human primates before. The developmental expression trajectory of prelimbic DAGL $\alpha$ and MGLL reported here agrees with the study by Subbanna et al. describing a similar increase and stabilisation of DAGL $\alpha$ and MGLL protein expression in the neocortical tissue of C57BL/6J mice across postnatal development (41). DAGL $\alpha$ protein expression increased from PND 2 and peaked at PND 15 in the neocortex, while MAGL protein expression increased from PND 2 and peaked at PND 45 (41). These peaks in 2-AG metabolic enzyme mRNA levels may represent periods of high endogenous cannabinoid system activity during postnatal brain development (42).

Importantly, our mouse DAGL $\alpha$ and MGLL mRNA expression profiles show similarities with human trajectories. We observed pronounced early postnatal increases in DAGL $\alpha$ and ABHD6 mRNA expression in the prelimbic cortex between PND 7 and 35 , which decreased slightly and then stabilised after this age. Mouse PND 7-35 corresponds with a human age range of infant to 11-year-old children (43), and this pattern of DAGL $\alpha$ and ABHD6 mRNA expression is largely consistent with expression patterns in human DLPFC tissue (17). Furthermore, a reduction in MGLL mRNA expression after puberty occurs in humans in the DLPFC (17), which we also observed in mice in the prelimbic cortex ( $c f$. PND 35 vs. PND 161). This may suggest similar regulation of these enzymes across development in the two species and is important when evaluating the validity of mouse model research in this context. It is possible that the increase in enzyme mRNA expression in early postnatal life reflects the involvement of the endocannabinoid system in neuronal growth and migration $(44,45)$, while its stabilisation after PND 14 (hippocampus) or
PND 35 (prelimbic cortex) may represent a switch to mediating retrograde signalling, whereby chemical messengers released from the postsynaptic cell bind to the presynaptic cell to mediate cell signalling. The involvement of the endocannabinoid system in retrograde signalling then appears to remain unchanged for the rest of postnatal life (46), which may be reflected in the reduction and stabilisation of these transcripts after PND 14 or 35.

Our findings suggest different rates of endocannabinoid system maturation of the prelimbic cortex and the hippocampus. The earlier peak in the hippocampus, compared to the prelimbic cortex, may indicate a longer period of maturation of the latter region (47). Indeed, the role of the endocannabinoid system in the stabilisation of cortical excitatory synapses, the balancing excitatory and inhibitory neurotransmission, and strengthening or elimination of excitatory cortical synapses during adolescence (48) support the extended maturation of this system in the prelimbic cortex, compared to the hippocampus. It is possible that fluctuations in endocannabinoid gene expression in early postnatal development (i.e., up to PND 14 or 35), which corresponds with early adolescence in humans, indicate a period during which this system is more susceptible to cannabinoid-induced developmental disruption, which may also increase the risk of cannabinoid-induced psychosis.

There were no differences in DAGL $\alpha$, MGLL, or ABHD6 mRNA expression in Nrg1 TM HET mice across development, compared to WT mice. This is the first study investigating these markers across development in a genetic mouse model for schizophrenia. Evidence for altered DAGL $\alpha$, MGLL, or ABHD6 mRNA expression in schizophrenia is mixed: elevated ABHD6 mRNA expression in PFC (Brodmann area 9) has been observed in patients with an illness duration of less than 15 years (49), whereas no differences in MGLL and DAGL $\alpha$ mRNA expression were found in a different cortical region, the DLPFC (Brodmann area 46) of patients with schizophrenia (50). In both of these studies, it is unknown whether these patients carried NRG1 risk alleles. It is possible that changes in 2-AG enzyme function may play a role in the development of schizophrenia-related phenotypes; however, this does not appear to depend on mutation in the NRG1 TM gene.

Heterozygous transmembrane domain Nrg1 mutant mice exhibit altered sensitivity to cannabinoid compounds (23-28), which, based on this study, appears unrelated to changes in the hippocampal or prelimbic cortical $\mathrm{CB}_{1}$ or $2-\mathrm{AG}$ enzyme mRNA expression levels. It is possible that alterations to the endocannabinoid system are present only in Nrg1 TM HET mice following a cannabinoid challenge to this system [e.g., Ref. $(27,28)]$. Alternatively, alternate signalling pathways and/or receptor systems may be responsible for cannabinoid-induced behavioural and neural changes in Nrg1 TM HET mice. Nrg1 TM HET mutation may cause alterations to other neurotransmitter systems that interact with the cannabinoid system, thereby mediating increased cannabinoid sensitivity. For example, serotonin $2 \mathrm{~A}$ receptors $\left(5-\mathrm{HT}_{2 \mathrm{~A}}\right)$ and $\mathrm{CB}_{1}$ receptors form heteromers, and crossantagonise each other at a receptor level to mediate behavioural effects of THC $(51,52)$. Importantly, our own work shows that Nrg1 TM HET mice exhibit some region-dependent reductions in $5-\mathrm{HT}_{2 \mathrm{~A}}$ receptor binding $(27,28)$, but increased $5-\mathrm{HT}_{2 \mathrm{~A}}$ binding across the striatum and frontal cortex (53). Thus, it is possible 
that altered $5-\mathrm{HT}_{2 \mathrm{~A}}$ receptor binding may affect how cannabinoid compounds bind to $\mathrm{CB}_{1} \mathrm{R}$ and thereby change the behavioural response of Nrg1 TM HET mice to cannabinoid treatment.

Alternatively, $\mathrm{CB}_{1}$ receptors may interact with $N$-methylD-aspartate (NMDA) receptors to modulate responses to cannabinoids in Nrg1 TM HET mice. $\mathrm{CB}_{1}$ and NMDA receptors interact to regulate cortical excitatory signalling (54). Also, adolescent THC treatment elevates NMDA receptor expression in the hippocampus, as well as auditory and insular cortices in Nrg1 TM HET, but not WT mice, supporting potential interactions between glutamatergic and cannabinoid systems in Nrgl mutant mice. Considering the dynamic nature of NMDA receptor development [e.g., changes in NMDA receptor expression on fast-spiking interneurons during cortical development (55) and elevated $c$-fos expression following NMDA receptor antagonist MK801 treatment, during early, but not late adolescence (56)], it is possible that baseline NMDA receptor expression may be altered in Nrg1 TM HET mice; this may affect $\mathrm{CB}_{1}$ receptor signalling to modulate behavioural and neural responses to cannabinoids in these mice.

In conclusion, this study demonstrates dynamic postnatal regulation of transcripts encoding key molecules of the endocannabinoid system. Our detailed mRNA expression profile of 2-AG enzymes is in line with the idea that the endocannabinoid system changes as the brain develops. Critically, these data highlight potential key postnatal time points where endocannabinoid system maturation may be susceptible to environmental insults, which could influence the development of mental illness. The mRNA transcripts examined were unaltered in Nrg1 TM HET mice, suggesting that these mice may not represent a model of altered endocannabinoid development.

\section{ETHICS STATEMENT}

Research and animal care procedures were approved by the University of New South Wales Animal Care and Ethics Committee

\section{REFERENCES}

1. Bayer TA, Falkai P, Maier W. Genetic and non-genetic vulnerability factors in schizophrenia: the basis of the "two hit hypothesis". J Psychiatr Res (1999) 33(6):543-8. doi:10.1016/S0022-3956(99)00039-4

2. Rapoport JL, Addington AM, Frangou S, Psych MR. The neurodevelopmental model of schizophrenia: update 2005. Mol Psychiatry (2005) 10(5):434-49. doi:10.1038/sj.mp.4001642

3. Karl T, Arnold JC. Schizophrenia: a consequence of gene-environment interactions? Front Behav Neurosci (2014) 8:435. doi:10.3389/fnbeh.2014.00435

4. Henquet C, Murray R, Linszen D, van Os J. The environment and schizophrenia: the role of cannabis use. Schizophr Bull (2005) 31(3):608-12. doi:10.1093/ schbul/sbi027

5. Large M, Sharma S, Compton MT, Slade T, Nielssen O. Cannabis use and earlier onset of psychosis: a systematic meta-analysis. Arch Gen Psychiatry (2011) 68(6):555-61. doi:10.1001/archgenpsychiatry.2011.5

6. Power RA, Verweij KJ, Zuhair M, Montgomery GW, Henders AK, Heath AC, et al. Genetic predisposition to schizophrenia associated with increased use of cannabis. Mol Psychiatry (2014) 19(11):1201-4. doi:10.1038/mp. 2014.51

7. Stefansson H, Sigurdsson E, Steinthorsdottir V, Bjornsdottir S, Sigmundsson T, Ghosh S, et al. Neuregulin 1 and susceptibility to schizophrenia. Am J Hum Genet (2002) 71(4):877-92. doi:10.1086/342734
(ACEC) in accordance with the Australian Code of Practice for the Care and Use of Animals for Scientific Purposes (ACEC approval number: 10/98B). The protocol was approved by the University of New South Wales Animal Care and Ethics Committee.

\section{AUTHOR CONTRIBUTIONS}

LL, RC, CW, and TK designed the research; LL performed the research; RC completed the data analysis and wrote the manuscript; and LL, RC, CW, and TK revised the manuscript and approved it prior to submission.

\section{ACKNOWLEDGMENTS}

We thank Shan Yuan Tsai-Chin for technical assistance, Adam Bryan for animal care and Kevin Taylor at Australian BioResources for the provision of facilities for tissue collection. We would also like to thank Jerry Tanda for critical comments on the manuscript.

\section{FUNDING}

This work was supported by the Schizophrenia Research Institute (utilising infrastructure funding from the NSW Ministry of Health and the Macquarie Group Foundation), the University of New South Wales and Neuroscience Research Australia. TK was supported by a Career Development Fellowship (Level 2) from the National Health and Medical Research Council (NHMRC: $\# 1045643)$, a NHMRC project grant (\#1102012), the NHMRC dementia research team initiative (\#1095215), and the Rebecca L. Cooper Medical Research Foundation Ltd.

\section{SUPPLEMENTARY MATERIAL}

The Supplementary Material for this article can be found online at http://www.frontiersin.org/articles/10.3389/fpsyt.2018.00011/ full\#supplementary-material.

8. Han S, Yang BZ, Kranzler HR, Oslin D, Anton R, Farrer LA, et al. Linkage analysis followed by association show NRG1 associated with cannabis dependence in African Americans. Biol Psychiatry (2012) 72(8):637-44. doi:10.1016/ j.biopsych.2012.02.038

9. Li B, Woo RS, Mei L, Malinow R. The neuregulin-1 receptor erbB4 controls glutamatergic synapse maturation and plasticity. Neuron (2007) 54(4):583-97. doi:10.1016/j.neuron.2007.03.028

10. Newell KA, Karl T, Huang XF. A neuregulin 1 transmembrane domain mutation causes imbalanced glutamatergic and dopaminergic receptor expression in mice. Neuroscience (2013) 248:670-80. doi:10.1016/j.neuroscience.2013.06.037

11. Keimpema E, Mackie K, Harkany T. Molecular model of cannabis sensitivity in developing neuronal circuits. Trends Pharmacol Sci (2011) 32(9):551-61. doi:10.1016/j.tips.2011.05.004

12. Leweke FM, Giuffrida A, Wurster U, Emrich HM, Piomelli D. Elevated endogenous cannabinoids in schizophrenia. Neuroreport (1999) 10(8):1665-9. doi:10.1097/00001756-199906030-00008

13. Giuffrida A, Leweke FM, Gerth CW, Schreiber D, Koethe D, Faulhaber J, et al. Cerebrospinal anandamide levels are elevated in acute schizophrenia and are inversely correlated with psychotic symptoms. Neuropsychopharmacology (2004) 29(11):2108-14. doi:10.1038/sj.npp.1300558

14. Eggan SM, Hashimoto T, Lewis DA. Reduced cortical cannabinoid 1 receptor messenger RNA and protein expression in schizophrenia. Arch Gen Psychiatry (2008) 65(7):772-84. doi:10.1001/archpsyc.65.7.772 
15. Eggan SM, Stoyak SR, Verrico CD, Lewis DA. Cannabinoid CB1 receptor immunoreactivity in the prefrontal cortex: comparison of schizophrenia and major depressive disorder. Neuropsychopharmacology (2010) 35(10):2060-71. doi:10.1038/npp.2010.75

16. Dalton VS, Long LE, Weickert CS, Zavitsanou K. Paranoid schizophrenia is characterized by increased CB1 receptor binding in the dorsolateral prefrontal cortex. Neuropsychopharmacology (2011) 36(8):1620-30. doi:10.1038/ npp.2011.43

17. Long LE, Lind J, Webster M, Weickert CS. Developmental trajectory of the endocannabinoid system in human dorsolateral prefrontal cortex. $B M C$ Neurosci (2012) 13:87. doi:10.1186/1471-2202-13-87

18. Walss-Bass C, Liu W, Lew DF, Villegas R, Montero P, Dassori A, et al. A novel missense mutation in the transmembrane domain of neuregulin 1 is associated with schizophrenia. Biol Psychiatry (2006) 60(6):548-53. doi:10.1016/j. biopsych.2006.03.017

19. Karl T, Duffy L, Scimone A, Harvey RP, Schofield PR. Altered motor activity, exploration and anxiety in heterozygous neuregulin 1 mutant mice: implications for understanding schizophrenia. Genes Brain Behav (2007) 6(7):677-87. doi:10.1111/j.1601-183X.2006.00298.x

20. O’Tuathaigh CM, Babovic D, O’Sullivan GJ, Clifford JJ, Tighe O, Croke DT, et al. Phenotypic characterization of spatial cognition and social behavior in mice with 'knockout' of the schizophrenia risk gene neuregulin 1. Neuroscience (2007) 147(1):18-27. doi:10.1016/j.neuroscience.2007.03.051

21. Duffy L, Cappas E, Lai D, Boucher AA, Karl T. Cognition in transmembrane domain neuregulin 1 mutant mice. Neuroscience (2010) 170(3):800-7. doi:10.1016/j.neuroscience.2010.07.042

22. Chesworth R, Downey L, Logge W, Killcross S, Karl T. Cognition in female transmembrane domain neuregulin 1 mutant mice. Behav Brain Res (2012) 226(1):218-23. doi:10.1016/j.bbr.2011.09.019

23. Boucher AA, Arnold JC, Duffy L, Schofield PR, Micheau J, Karl T. Heterozygous neuregulin 1 mice are more sensitive to the behavioural effects of Delta9tetrahydrocannabinol. Psychopharmacology (Berl) (2007) 192(3):325-36. doi:10.1007/s00213-007-0721-3

24. Boucher AA, Hunt GE, Karl T, Micheau J, McGregor IS, Arnold JC. Heterozygous neuregulin 1 mice display greater baseline and Delta(9)tetrahydrocannabinol-induced c-Fos expression. Neuroscience (2007) 149(4):861-70. doi:10.1016/j.neuroscience.2007.08.020

25. Long LE, Chesworth R, Arnold JC, Karl T. A follow-up study: acute behavioural effects of Delta(9)-THC in female heterozygous neuregulin 1 transmembrane domain mutant mice. Psychopharmacology (Berl) (2010) 211(3):277-89. doi:10.1007/s00213-010-1896-6

26. Boucher AA, Hunt GE, Micheau J, Huang X, McGregor IS, Karl T, et al. The schizophrenia susceptibility gene neuregulin 1 modulates tolerance to the effects of cannabinoids. Int J Neuropsychopharmacol (2011) 14(5):631-43. doi:10.1017/S146114571000091X

27. Long LE, Chesworth R, Huang XF, Wong A, Spiro A, McGregor IS, et al. Distinct neurobehavioural effects of cannabidiol in transmembrane domain neuregulin 1 mutant mice. PLoS One (2012) 7(4):e34129. doi:10.1371/journal. pone.0034129

28. Long LE, Chesworth R, Huang XF, McGregor IS, Arnold JC, Karl T. Transmembrane domain Nrg1 mutant mice show altered susceptibility to the neurobehavioural actions of repeated THC exposure in adolescence. Int J Neuropsychopharmacol(2013) 16(1):163-75.doi:10.1017/S1461145711001854

29. Sherif M, Radhakrishnan R, D'Souza DC, Ranganathan M. Human laboratory studies on cannabinoids and psychosis. Biol Psychiatry (2016) 79(7):526-38. doi:10.1016/j.biopsych.2016.01.011

30. Marsicano G, Lutz B. Expression of the cannabinoid receptor CB1 in distinct neuronal subpopulations in the adult mouse forebrain. Eur J Neurosci (1999) 11(12):4213-25. doi:10.1046/j.1460-9568.1999.00847.x

31. Lewis DA. Cortical circuit dysfunction and cognitive deficits in schizophrenia - implications for preemptive interventions. Eur J Neurosci (2012) 35(12):1871-8. doi:10.1111/j.1460-9568.2012.08156.x

32. Owen MJ, Sawa A, Mortensen PB. Schizophrenia. Lancet (2016) 388:86-97. doi:10.1016/S0140-6736(15)01121-6

33. Karl T, Arnold JC. What does a mouse tell us about neuregulin 1-cannabis interactions? Front Cell Neurosci (2013) 7:18. doi:10.3389/fncel.2013.00018

34. Berrendero F, Sepe N, Ramos JA, Di Marzo V, Fernandez-Ruiz JJ. Analysis of cannabinoid receptor binding and mRNA expression and endogenous cannabinoid contents in the developing rat brain during late gestation and early postnatal period. Synapse (1999) 33(3):181-91. doi:10.1002/ (SICI) 1098-2396(19990901)33:3<181:AID-SYN3>3.0.CO;2-R

35. Wang X, Dow-Edwards D, Keller E, Hurd YL. Preferential limbic expression of the cannabinoid receptor mRNA in the human fetal brain. Neuroscience (2003) 118(3):681-94. doi:10.1016/S0306-4522(03)00020-4

36. Danglot L, Triller A, Marty S. The development of hippocampal interneurons in rodents. Hippocampus (2006) 16(12):1032-60. doi:10.1002/hipo.20225

37. Berrendero F, Garcia-Gil L, Hernandez ML, Romero J, Cebeira M, de Miguel R, et al. Localization of mRNA expression and activation of signal transduction mechanisms for cannabinoid receptor in rat brain during fetal development. Development (1998) 125(16):3179-88.

38. Eggan SM, Mizoguchi Y, Stoyak SR, Lewis DA. Development of cannabinoid 1 receptor protein and messenger RNA in monkey dorsolateral prefrontal cortex. Cereb Cortex (2010) 20(5):1164-74. doi:10.1093/cercor/bhp179

39. Lewis DA, Hashimoto T, Morris HM. Cell and receptor type-specific alterations in markers of GABA neurotransmission in the prefrontal cortex of subjects with schizophrenia. Neurotox Res (2008) 14(2-3):237-48. doi:10.1007/ BF03033813

40. Verdurand M, Fillman SG, Weickert CS, Zavitsanou K. Increases in $[3 \mathrm{H}]$ muscimol and $[3 \mathrm{H}]$ flumazenil binding in the dorsolateral prefrontal cortex in schizophrenia are linked to alpha4 and gamma2S mRNA levels respectively. PLoS One (2013) 8(1):e52724. doi:10.1371/journal.pone.0052724

41. Subbanna S, Psychoyos D, Xie S, Basavarajappa BS. Postnatal ethanol exposure alters levels of 2-arachidonylglycerol-metabolizing enzymes and pharmacological inhibition of monoacylglycerol lipase does not cause neurodegeneration in neonatal mice. J Neurochem (2015) 134(2):276-87. doi:10.1111/ jnc. 13120

42. Harkany T, Mackie K, Doherty P. Wiring and firing neuronal networks: endocannabinoids take center stage. Curr Opin Neurobiol (2008) 18(3):338-45. doi:10.1016/j.conb.2008.08.007

43. Semple BD, Blomgren K, Gimlin K, Ferriero DM, Noble-Haeusslein LJ. Brain development in rodents and humans: identifying benchmarks of maturation and vulnerability to injury across species. Prog Neurobiol (2013) 106-107:1-16. doi:10.1016/j.pneurobio.2013.04.001

44. Diaz-Alonso J, Guzman M, Galve-Roperh I. Endocannabinoids via CB(1) receptors act as neurogenic niche cues during cortical development. Philos Trans R Soc Lond B Biol Sci (2012) 367(1607):3229-41. doi:10.1098/ rstb.2011.0385

45. Gaffuri AL, Ladarre D, Lenkei Z. Type-1 cannabinoid receptor signaling in neuronal development. Pharmacology (2012) 90(1-2):19-39. doi:10.1159/000339075

46. Oudin MJ, Hobbs C, Doherty P. DAGL-dependent endocannabinoid signalling: roles in axonal pathfinding, synaptic plasticity and adult neurogenesis. Eur J Neurosci (2011) 34(10):1634-46. doi:10.1111/j.1460-9568. 2011.07831.x

47. Schubert D, Martens GJ, Kolk SM. Molecular underpinnings of prefrontal cortex development in rodents provide insights into the etiology of neurodevelopmental disorders. Mol Psychiatry (2015) 20(7):795-809. doi:10.1038/ mp. 2014.147

48. Meyer HC, Lee FS, Gee DG. The role of the endocannabinoid system and genetic variation in adolescent brain development. Neuropsychopharmacology (2018) 43(1):21-33. doi:10.1038/npp.2017.143

49. Volk DW, Siegel BI, Verrico CD, Lewis DA. Endocannabinoid metabolism in the prefrontal cortex in schizophrenia. Schizophr Res (2013) 147(1):53-7. doi:10.1016/j.schres.2013.02.038

50. Volk DW, Eggan SM, Lewis DA. Alterations in metabotropic glutamate receptor lalpha and regulator of $\mathrm{G}$ protein signaling 4 in the prefrontal cortex in schizophrenia. Am J Psychiatry (2010) 167(12):1489-98. doi:10.1176/appi. ajp.2010.10030318

51. Franklin JM, Mathew M, Carrasco GA. Cannabinoid-induced upregulation of serotonin $2 \mathrm{~A}$ receptors in the hypothalamic paraventricular nucleus and anxiety-like behaviors in rats. Neurosci Lett (2013) 548:165-9. doi:10.1016/j. neulet.2013.05.039

52. Vinals X, Moreno E, Lanfumey L, Cordomi A, Pastor A, de La Torre R, et al. Cognitive impairment induced by Delta9-tetrahydrocannabinol occurs through heteromers between cannabinoid CB1 and serotonin 5-HT2A receptors. PLoS Biol (2015) 13(7):e1002194. doi:10.1371/journal.pbio.1002194 
53. Dean B, Karl T, Pavey G, Boer S, Duffy L, Scarr E. Increasedlevels of serotonin $2 A$ receptors and serotonin transporter in the CNS of neuregulin 1 hypomorphic/ mutant mice. Schizophr Res (2008) 99(1-3):341-9. doi:10.1016/j.schres. 2007.10.013

54. Sanchez-Blazquez P, Rodriguez-Munoz M, Garzon J. The cannabinoid receptor 1 associates with NMDA receptors to produce glutamatergic hypofunction: implications in psychosis and schizophrenia. Front Pharmacol (2014) 4:169. doi:10.3389/fphar.2013.00169

55. Wang HX, Gao WJ. Cell type-specific development of NMDA receptors in the interneurons of rat prefrontal cortex. Neuropsychopharmacology (2009) 34(8):2028-40. doi:10.1038/npp.2009.20

56. Inta I, Domonkos E, Pfeiffer N, Sprengel R, Bettendorf M, Lang UE, et al. Puberty marks major changes in the hippocampal and cortical c-Fos activation pattern induced by NMDA receptor antagonists. Neuropharmacology (2017) 112(Pt A):181-7. doi:10.1016/j.neuropharm.2016.03.023

Conflict of Interest Statement: The research was conducted in the absence of any commercial or financial relationships that could be construed as a potential conflict of interest.

Copyright (c) 2018 Chesworth, Long, Weickert and Karl. This is an open-access article distributed under the terms of the Creative Commons Attribution License (CC BY). The use, distribution or reproduction in other forums is permitted, provided the original author(s) and the copyright owner are credited and that the original publication in this journal is cited, in accordance with accepted academic practice. No use, distribution or reproduction is permitted which does not comply with these terms. 